\title{
CASE REPORT OF AN INCIDENTAL UNICENTRIC CASTLEMAN DISEASE
}

\author{
Elif Cengiz ${ }^{1} \odot$, Alperen Taha Certel ${ }^{1} \odot$, Mert Yücel Ayrık ${ }^{1} \odot$, Mehmet Gürkan Arıkan² $\odot$, \\ Elif Mercan Demirtaş ${ }^{3}$, Fulya Öz Puyan ${ }^{3} \mathbb{C}$, İrfan Hüseyin Atakan² ${ }^{\circledR}$
}

${ }^{1}$ Trakya University School of Medicine, Edirne, TURKEY

${ }^{2}$ Department of Urology, Trakya University School of Medicine, Edirne, TURKEY

${ }^{3}$ Department of Pathology, Trakya University School of Medicine, Edirne, TURKEY

\begin{abstract}
Aims: To emphasize the hardship of diagnosing Castleman disease and present a potential treatment method. Case Report: A sixty-three-year-old male patient was admitted to the outpatient clinic with an attack of acute cholecystitis. The patient's abdominal computed tomography revealed an incidentally detected lipid dense solid mass $(64 \times 53 \times 37 \mathrm{~mm})$ at the level of right renal hilum with 29x13 mm solid components in the middle. The patient was admitted to the urology department and underwent surgery where the mass was totally excised due to suspicion of a malignancy (liposarcoma). Histopathological examination later on resulted with unicentric Castleman disease, hyaline vascular subtype. Conclusion: Since unicentric Castleman disease has an asymptomatic clinical course and is quite rare, it is necessary to rule out many potential possibilities before reaching a proper diagnosis. However, unicentric Castleman disease usually exhibits a good prognosis after the removal of the affected lymph node. Still, Castleman disease should be a candidate considered in the differential diagnosis of patients with incidentally discovered lymphadenopathy. On the whole, for a better understanding of underlying pathophysiology, there still lies a gap to be filled with knowledge acquired through further studies. Keywords: Castleman disease, lymphoproliferative disorder, lymphadenopathy
\end{abstract}

\section{INTRODUCTION}

First described by Benjamin Castleman in 1954 as a rare lymphoproliferative disorder, Castleman disease (CD) can be separated into two main groups based on the number of lymph nodes involved: unicentric Castleman disease (UCD) and multicentric Castleman disease (MCD) (1). While UCD affects only a single lymph node or lymph node chain, MCD is a more widespread disease (2). Lymph nodes of the trunk, neck, and abdomen are affected by 70,15 , and $15 \%$, respectively. Additionally, there have been cases reported with affected lung, larynx, parotid, pancreas, and meninges (3). A study aiming to elucidate the incidence rate of CD found the rate to be 21-25 per million persons per year, with UCD being the most common type (4). Thus, the etiology of the disease has not been fully understood due to its rarity and clinical heterogeneity. The five-year overall survival rate is $91 \%$ in UCD (5). Diagnosis is made with the biopsy of the affected lymph node (5).

Histological classification of CD is characterized with 3 variants: hyaline vascular (HV), the plasma-cell, and the mixed form variant (2). Unicentric presentation most commonly demonstrates $\mathrm{HV}$, whereas MCD presents plasma-cell or mixed variant more (6). $\mathrm{CD}$ is usually associated with increased production of interleukin-6 (IL-6), where the plasma-cell variant has been linked with human immunodeficiency virus (HIV) and human herpesvirus 8 $(\mathrm{HHV}-8)(1,2)$. Although different therapeutic approaches are defined for the diagnosis and treatment of UCD, excision is still the gold standard (7).
By presenting this rare case of UCD we aim to raise an awareness of CD's inclusion as an entity in differential diagnosis with patients who have single or more lymphadenopathy and put forward a potential treatment method.

\section{CASE REPORT}

A sixty-three-year-old male patient was admitted to the outpatient clinic of Trakya University Hospital with an attack of acute cholecystitis. In the patient's past medical history, he had a diagnosis of hypertension. In the outpatient clinic examination, the patient's onset blood pressure, heart rate, and body temperature were normal, whereas he had gastrointestinal symptoms and weight loss. His physical examination revealed there was no bilateral costovertebral angle sensitivity. The routine hemogram, urinalysis, and serum electrolytes were normal. Ultrasonography was performed in the emergency department and no stones were found in the gallbladder. After the USG, a computed tomography (CT) scan was performed as well. In the patient's abdominal CT scan an incidental lipid dense mass of $64 \times 53 \times 37 \mathrm{~mm}$ was detected at the right renal hilum level with $29 \times 13 \mathrm{~mm}$ solid components in the center (Figure 1).

Liposarcoma had been considered in the differential diagnosis and the total excision of the mass had been planned for the exact diagnosis and treatment. The patient was operated on and followed by the urology department. The operation was performed with an anterior subcostal incision. After the colon was medialized, the re- 
nal vein and artery were reached while protecting the duodenum. Mass was later on excised due to a suspicion of malignancy. Postoperative follow-up was uneventful and hematological blood tests were found to be normal.

Liposarcoma and primary renal cell carcinoma (hypercalcemia, fever, and erythrocytosis were not present) were considered in the differential diagnosis. Total excision of the mass has been planned both for the exact diagnosis and treatment. The patient was admitted to the hospital and later was operated on by the urology department. The operation was performed with an anterior subcostal incision. After the colon was medialized, the renal vein and artery were reached while protecting the duodenum. The mass was excised due to a malignancy suspicion. The cholecystectomy was also performed during the operation. The postoperative follow-ups were done by the hematology department. The follow-up was uneventful and hematological blood tests were found to be normal.
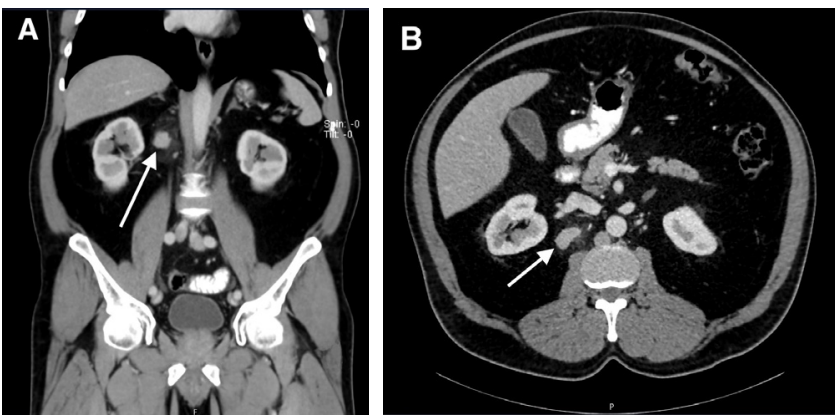

Figure 1: A: Coronal plane of abdominal CT scan (lipid dense mass shown with the arrow). B: Transverse plane of abdominal CT scan (lipid dense mass shown with the arrow).
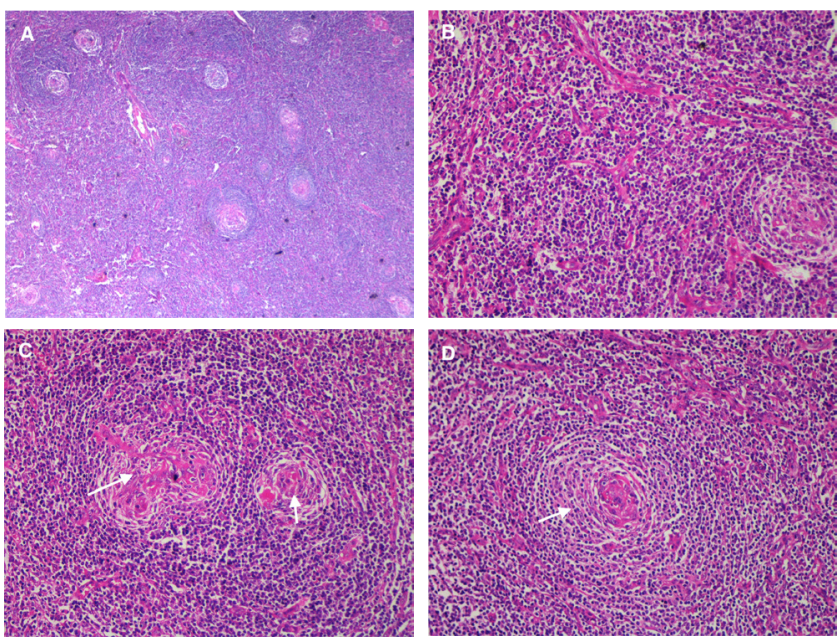

Figure 2: A: Involuted lymphoid follicles distributed in lymph node parenchyma and vascularized structures with hyalinized walls aligned with hyperplastic endothelium in the interfollicular zone (H\&E, x100). B: Incrementation of hyalinized vascular structures created by hyperplastic endothelium in interfollicular spaces and regressing lymphoid follicle on the right (H\&E, x400). C: The view of Castleman disease's characteristic structure "Lollipop follicle" shown with the white arrows, atrophic germinal center, and hyalinized small vein of lymphoid follicle (H\&E, x400). D: The typical "onion skin pattern" view (Arrow), which is common to be seen in hyalinized germinal center together with concentrated layers of small uniformed Mantle zone lymphocytes (H\&E, x400).
Following patient's surgery, the specimen was sent to the pathology department for further analysis. Histopathological examination resulted with $\mathrm{CD}$, hyaline vascular subtype. The HHV-8 test, which was performed to determine the type of $\mathrm{CD}$, was negative. In addition, B lymphocyte markers were checked in order to exclude lymphoma. The immunohistochemical analysis results were as follows: mantle zone lymphocytes were stained with the IgM, OCT-2 and PAX5 stained the atrophic follicules, MUM1 stained the plasma cells and the BCL- 6 test was positive at the atrophic germinal centers. Characteristic HV histological patterns such as involuted lymphoid follicles, incrementation of hyalinized vascular structures, lollipop follicle and onion skin pattern on the hyalinized germinal center were found (Figure 2). In addition, positive staining for CD20 in germinal center (Figure 3A), positive staining for CD21 antibody of dendritic cell population in germinal centers (Figure 3B) and positive staining for CD 3 antibody in T lymphocytes around germinal centers (Figure 3C) were seen.

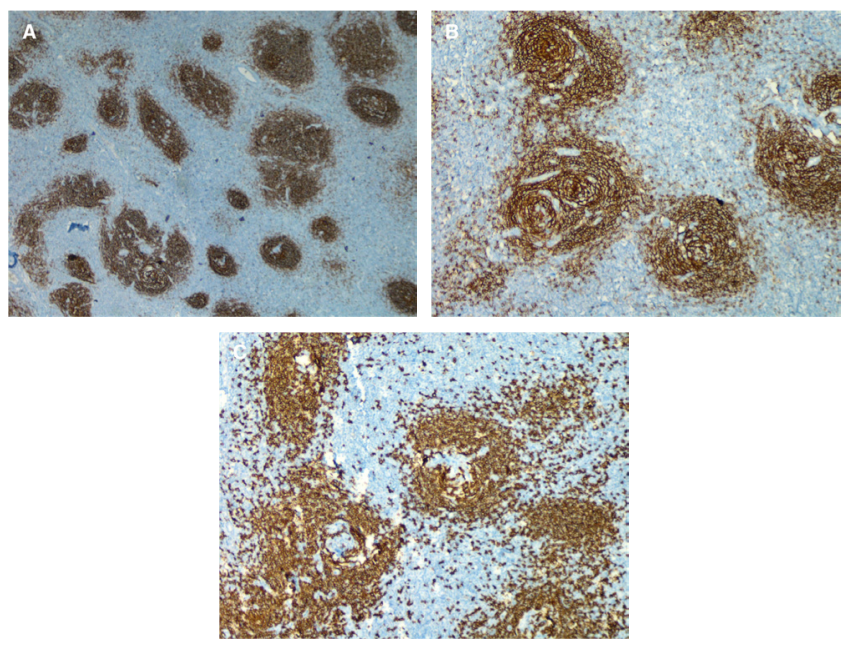

Figure 3: Immunohistochemical analysis. A: Positive staining for CD20 antibody in germinal center of different sizes (magnification x100). B: Positive staining for CD21 antibody of dendritic cell population in germinal centers with locally clinging patterns (magnification x200). C: Positive staining for CD 3 antibody in T lymphocytes around germinal centers (magnification $x 200$ ).

\section{DISCUSSION}

Since UCD is often asymptomatic, early diagnosis may not always be possible. In some cases, especially the ones with retroperitoneal masses, tumors may have different appearances, which makes the pre-operative diagnosis exceedingly difficult (8). An enlarged lymph node can be detected incidentally during a physical examination or imaging test performed for another reason. Nakata et al. (9) presented a 47-year-old with a mass in pelvic retroperitoneum, incidentally diagnosed case with no systemic symptoms such as pelvic pain, fatigue, fever, or weight loss. In addition, the patient's serum levels of carcinoembryonic antigen (CEA), cancer antigen (CA) 19-9, CA125, and interleukin 6 (IL-6) were within the normal range. While the mass is usually detected incidentally, as Nakata et al. (9) reported, it can also manifest itself with specific symptoms caused by the effect of the mass or adjacent organs (10, 11). There have been individuals reported with palpable masses and dyspeptic symptoms $(10,11)$. Hence, these studies indicate how complicated the diagnosis of CD can be. 
In our case, a lipid dense solid was incidentally detected at the level of the right renal artery on CT, performed during an attack of cholecystitis. Patients with acute cholecystitis usually present with symptoms of unremitting right upper quadrant pain, anorexia, nausea, vomiting, and fever as in our case (12). Gallbladder wall thickening, pericholecystic fluid, or a sonographic Murphy's sign are among the typical diagnostic findings (13).

The clinical presentation of CD is usually associated with autoimmune and infectious diseases. Excessive production of IL-6 and a viral analog of IL-6 are important factors of pathogenesis (2). Moreover, the plasma-cell variant is associated with HIV and human herpesvirus 8 (HHV-8) infections (1). Our case was HHV-8 negative, thus plasma-cell variant possibility was excluded.

There are mainly two subtypes of UCD hyaline vascular variant and the plasma-cell variant. The HV variant's histological pattern is usually diagnostic with multiple tight aggregates of follicular dendritic cells or atrophic follicles (1). On the other hand, the plasma cell variants of CD are less-defined histologically (1). Our patient's $\mathrm{CD}$ was an $\mathrm{HV}$ variant, thus some characteristic histological patterns such as involuted lymphoid follicles (Figure 2A), incrementation of hyalinized vascular structures (Figure 2B), hyalinized arteriole characterized with lollipop follicle (Figure 2C) and onion skin pattern on the hyalinized germinal center (Figure 2D) were seen clearly.

Treatment response is evaluated by normalization of previous clinical/laboratory norms after lymph nodes are completely removed (7). Corticosteroids and rituximab or adjuvant radiotherapy are the preferred options in reducing tumors too large for removal (14). Although radiotherapy can be applied to patients who cannot be operated due to comorbidities or residual tumor remains, surgical excision of the mass should still remain as the primary treatment option (14). For this reason, the medical team should carefully scrutinize all aspects and risk factors before reaching a consensus for the treatment.

In conclusion, various other possibilities are ruled out first on the path of diagnosing CD. The reason being that it is asymptomatic in most cases and is extremely rare. Despite that, UCD patients usually exhibit a good prognosis after the affected lymph node is excised. Therefore, surgical excision still remains an important and effective treatment option. Nonetheless, we hope that this case report will give physicians a better insight into this rare event.
Ethics Committee Approval: N/A

Informed Consent: Informed consent was obtained from the participants of this study.

Conflict of Interest: The authors declare no conflict of interest.

Author Contributions: Concept: EC, ATC, MYA, MGA, EM, FÖP, İHA. Design: EC, ATC, MYA, MGA, EM, FÖP, İHA. Supervision: EC, ATC, MYA, MGA, EM, FÖP, İHA. Resources: EC, ATC, MYA, MGA, EM, FÖP, İHA. Materials: EC, ATC, MYA, MGA, EM, FÖP, İHA. Data collection and/or Processing: EC, ATC, MYA, MGA, EM, FÖP, İHA. Analysis and/or Interpretation: EC, ATC, MYA, MGA, EM, FÖP, İHA. Literature Search: EC, ATC, MYA, MGA, EM, FÖP, İHA. Writing Manuscript: EC, ATC, MYA, MGA, EM, FÖP, İHA. Critical Review: EC, ATC, MYA, MGA, EM, FÖP, İHA.

Financial disclosure: The authors declared that this study received no financial support.

Editor-in-chief's Note: Three of the authors of this article, Elif Cengiz, Alperen Taha Certel, Mert Yücel Ayrik are members of the editorial board of Turkish Medical Student Journal. However, they did not take place at any stage of the editorial decision of the manuscript. The editors who evaluated this manuscript are from other institutions.

\section{REFERENCES}

1. Chan KL, Lade S, Prince HM et al. Update and new approaches in the treatment of Castleman disease. J Blood Med 2016;3(7):145-58.

2. Kaur H, Xiang Z, Kunthur A et al. Castleman disease. Fed Pract 2015;32(7):41-6.

3. Bonekamp D, Horton KM, Hruban RH et al. Castleman disease: The great mimic. RadioGraphics 2011;31:1793-807.

4. Munshi N, Mehra M, van de Velde $\mathrm{H}$ et al. Use of a claims database to characterize and estimate the incidence rate for Castleman disease. Leuk Lymphoma 2015;56(5):1252-60.

5. Dispenzieri A, Armitage JO, Loe MJ et al. The clinical spectrum of Castleman's disease. Am J Hematol 2012;87(11):997-1002.

6. Dispenzieri A, Gertz MA. Treatment of Castleman's disease. Curr Treat Options Oncol 2005;6(3):255-66.

7. Talat N, Belgaumkar AP, Schulte KM. Surgery in Castleman's disease: A systematic review of 404 published cases. Ann Surg 2012;255(4):677-84.

8. Nirhale DS, Bharadwaj RN, Athavale VS et al. Castleman's disease: A rare diagnosis in the retroperitoneum. Indian J Surg 2013;75(1):9-11.

9. Nakata K, Iwahashi N, Matsukawa H et al. Laparoscopically resected Castleman's disease in the pelvic retroperitoneum: A case report. Mol Clin Oncol 2020;12(2):169-73.

10. Abdessayed N, Bdioui A, Ammar H et al. Retroperitoneal unicentric Castleman's disease: A case report. Int J Surg Case Rep 2017;31:54-7.

11. Bracale U, Pacelli F, Milone M et al. Laparoscopic treatment of abdominal unicentric Castleman's disease: A case report and literature review. BMC Surg 2017;17(1):38.

12. American Cancer Society. Treatment of Localized (Unicentric) Castleman Disease (cited 2020 December 24) Available from: URL: https://www.cancer.org/ cancer/castleman-disease/treating/treating-localized.html. 